\title{
On the Usage of GRECOSAR, an Orbital Polarimetric SAR Simulator of Complex Targets, to Vessel Classification Studies
}

\author{
Gerard Margarit, Student Member, IEEE, Jordi J. Mallorqui, Member, IEEE, \\ Juan M. Rius, Member, IEEE, and Jesus Sanz-Marcos
}

\begin{abstract}
This paper presents a synthetic aperture radar (SAR) simulator that is able to generate polarimetric SAR (POLSAR) and polarimetric inverse SAR data of complex targets. It solves the electromagnetic problem via high-frequency approximations, such as physical optics and the physical theory of diffraction, with notable computational efficiency. In principle, any orbital monostatic sensor working at any band, resolution, and operating mode can be modeled. To make simulations more realistic, the target's bearing and speed are considered, and for the particular case of vessels, even the translational and rotational movements induced by the sea state. All these capabilities make the simulator a powerful tool for supplying large amounts of data with precise scenario information and for testing future sensor configurations. In this paper, the usefulness of the simulator on vessel classification studies is assessed. Several simulated polarimetric images are presented to analyze the potentialities of coherent target decompositions for classifying complex geometries, thus basing an operational algorithm. The limitations highlighted by the results suggest that other approaches, like POLSAR interferometry, should be explored.
\end{abstract}

Index Terms-Coherent target decompositions (CTD), polarimetric inverse SAR (POLISAR), polarimetric SAR (POLSAR), SAR image shifts, SAR simulation, ship classification, synthetic aperture radar (SAR).

\section{INTRODUCTION}

$\mathbf{N}$ OWADAYS, the European Community (EC) is devoting many efforts for developing efficient vessel monitoring systems (VMSs) able to control in a global scale the maritime traffic, especially fishing vessels, cruising the European waters. Their main goal is to help authorities achieve a better management of the surveillance resources in front of emergency situations or illegal fishing activities. Early monitoring systems have been based on transponders that when placed onboard vessels are able to provide vessel identification and position via satellite communications. A good example is the VMS [1]. Since January 1, 2005, all EC fishing vessels exceeding $15 \mathrm{~m}$ in overall length are subjected to VMS, excluding those that

Manuscript received May 12, 2005; revised June 19, 2006. This work was supported in part by the European Union under IMPAST Project Q5RS-200102266, the Spanish MCYT and EU FEDER under Project TIC2002-04451C02-01 and Project TEC2005-06863-C02-01, and the Spanish MECD under Grant AP2002-2313.

The authors are with the Department of Signal Theory and Communications, Universitat Politècnica de Catalunya, 08034 Barcelona, Spain (e-mail: gerardm@tsc.upc.edu; mallorqui@tsc.upc.edu).

Digital Object Identifier 10.1109/TGRS.2006.881120 are used exclusively for aquaculture and operating exclusively inside the baselines of Member States. Third-country vessels subject to VMS are obliged to have an operational satellite tracking device installed onboard when they are in EC waters. Despite transponder-based monitoring solutions providing accurate results, they are not perfectly suitable for monitoring illegal activities as they need specific equipment to be installed on each ship under control. The possibility that such hardware can be intentionally disconnected or damaged drops their overall autonomy and reduces its monitoring capability. A complementary or even alternative solution may be found on remote sensing instruments, particularly synthetic aperture radar (SAR). Besides the demanded independence, the capability to cover vast areas with reasonable resolutions in all weather conditions is an important advantage to be considered. To be aware of the real potentialities of this technology in vessel monitoring, the EC has promoted two projects, namely: 1) Improving fisheries Monitoring through integrating Passive and Active Satellite based Technologies (IMPAST) [2] and 2) Detection, Classification, and Identification of Marine Traffic from Space (DECLIMS) [3]. Both were defined to provide focus for research into the usage of satellite imagery for maritime vessel detection, classification, and identification. In addition, they tried to identify the advantages and drawbacks of different approaches, to strengthen the infrastructure capable of meeting the demands of users, and to help driving developments of new sensors and platforms toward the operational need of vessel monitoring.

While detection techniques with SAR imagery are quite well established, and some operational algorithms are available, classification is still an open issue. Single-polarization images present strong limitations toward a reliable classification, but coherent target decompositions (CTD) [4] applied to polarimetric SAR (POLSAR) data seem to be able to provide better results. These theorems express the scattering matrix as the complex sum of basis matrices, each of them associated to an elementary scattering mechanism. Such mechanisms can also be related to the geometrical shapes of the vessels' structure (like trihedrals, diplanes, etc.), allowing, in theory, the extraction of useful information for classification.

Anyway, up to now, it has not been fully confirmed that CTD is a reliable tool for ship classification. In addition to the difficulties on acquiring the required quantities of real data of vessels under different observation and environmental 
conditions with precise ground truth, the technological constraints of current sensors regarding resolution and polarimetric capabilities make it almost impossible to perform such a study. The only option to overcome this situation is the usage of numerical tools that reproduce in a computer the vessel SAR signature that a sensor would provide in a real and controlled scenario.

This paper presents a SAR simulator [5], [6] fulfilling these requirements. It has been developed at the Universitat Politècnica de Catalunya (UPC) in the framework of the IMPAST project and provides POLSAR and polarimetric inverse SAR (POLISAR) images of complex targets. The software can simulate sensors working at any orbit, carrier frequency, operating mode, and resolution, and to make the simulations more realistic, it can also consider the vessel's bearing, speed, and all movements induced by the sea state. These features are an important advantage in relation to current SAR simulation tools known by the authors. Certainly, most of them are focused on working with distributed targets [7]-[9] or to use different radar cross section (RCS) prediction tools [10], [11] to derive inverse SAR (ISAR) data. The former is oriented to ocean simulation and makes some simplifications as not considering diffraction and multiple scattering that would not properly work for complex targets. The latter is mainly designed to isolate scattering centers for military applications.

This paper is structured as follows. Section II presents a description of the SAR simulator as well as its validation. Its usefulness for classification studies is assessed in Section III, where simulated data sets processed for different targets and scenarios are presented and analyzed. The outlined conclusions point out the capabilities and limitations of POLSAR imagery for classification purposes as well as suggest new approaches to the problem.

\section{Simulator Description AND VAlidation}

The SAR simulator, known as graphical electromagnetic computation SAR (GRECOSAR) [5], [6], is a numerical tool that can generate POLSAR and POLISAR images of nondistributed complex targets for a wide range of flexible and perfectly controlled scenarios. Its core is formed by two software modules developed at UPC, namely: 1) the electromagnetic (EM) solver GRECO [12]-[14], which fixes most of the computational requirements, and 2) a SAR processor based on the extended chirp scaling algorithm (ECSA) [15].

The block scheme of the simulator is presented in Fig. 1. In this scheme, the dark gray boxes highlight the two main software modules, whereas the white ones highlight the different steps of simulation. The light gray boxes indicate the input parameters that define a simulation and used to calculate the orbital position of the platform, the antenna pointing and synthetic aperture length ("satellite parameters"), the chirp parameters ("radar parameters"), some environment-related information ("target environment parameters"), and the geometrical structure of the target in a GRECO-compatible format ("target model file"). Regarding the target environment, the main parameters are the sea state and the target's speed, bearing $(\beta)$, and position within the swath. This last parameter is

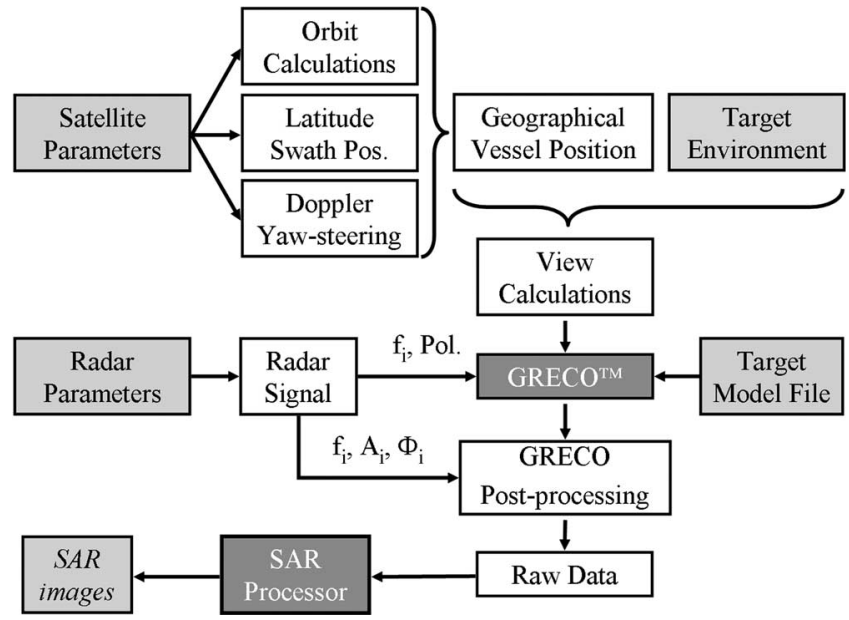

Fig. 1. Scheme of the SAR simulator.

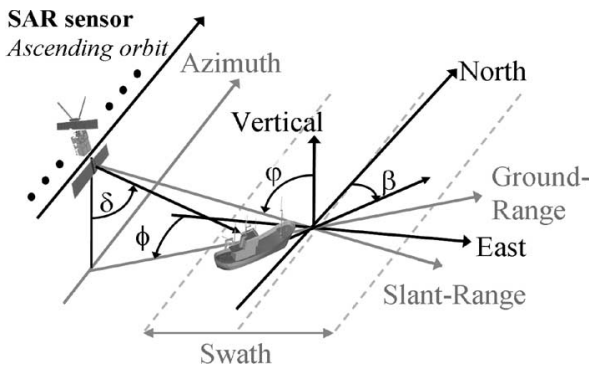

Fig. 2. Imaging geometry for SAR data. Despite the flat-Earth drawing, all calculations are carried out considering an ellipsoidal Earth.

fixed by the so-called near-far or look angle $(\delta)$, which is pictorially defined in the imaging geometry of SAR data shown in Fig. 2.

\section{A. Determination of the Satellite and Vessel Positions}

In the first step of a simulation, the sensor positions are calculated according to the orbit of the satellite, which is defined by their six Keplerian orbital elements. This process takes into account the so-called "yaw steering," which is used for most satellites to accommodate the high Doppler due to Earth rotation to the available pulse repetition frequency. The simulator can consider the problem in two different ways. The user can select either a yaw steering value from which the Doppler parameters are calculated or a Doppler centroid and adjust the required yaw steering for the given orbit, target latitude, and look angle. Once the yaw steering is known, the precise position of the target is calculated using the near-far angle $(\delta)$, the incidence angle $(\varphi)$, and the satellite positions.

\section{B. View Calculations}

The EM solver works as if the radar was placed in front of the computer screen where the object is visualized. This implies that the original vessel model has to be rotated in azimuth and elevation to be placed with the same orientation as it would be seen by the satellite. These rotations are controlled by the 


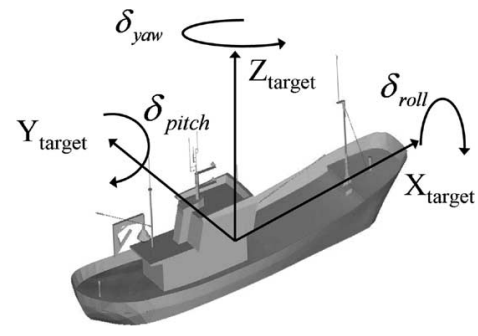

Fig. 3. Translational and rotational motions due to sea swell over the vessel model.

so-called "view angles," which are defined over a coordinate system fixed at the vessel's structure (see Fig. 3). They are computed by taking into account the target's position, bearing, and speed, the sea state, and the set of satellite positions in which the target is into the antenna beam.

Regarding target motions, cruising speed is simulated by modifying for each azimuth position the location of the target according to its bearing. On the other hand, vessel motions due to ocean waves are simulated by applying the proper translations (swaying, heaving, and surging) and rotations (pitch, roll, and yaw) to the vessel's axis. The former are assumed as additional linear motions, whereas the latter are defined by a set of angular velocities derived from the wave parameters (wave height, wave period, and wave direction relative to vessel's course) and vessel dimensions. In this process, a simple wave model that considers waves as sinusoids and computes the angular velocities without taking the hydrodynamic forces into account is used. This simplified model is enough for the simulator's purposes, mostly oriented to know the effects of vessel motions on SAR images rather than perfectly model a particular sea estate.

\section{Radar Signal Simulation}

The last step before starting the EM computations is the radar signal simulation. GRECO performs all calculations in the frequency domain, i.e., at a single frequency, while the simulation of SAR raw data is a time-domain problem. The chirp signal in time domain is emulated by using the discrete frequency values computed with a fast Fourier transform (FFT) over the temporal window in which the target is illuminated by the impinging radiation. The wider the target is on the range direction, the larger is the number of discrete frequencies to consider. In practice, due to the small target dimensions compared with the spatial chirp length, the number of frequencies can be a priori fixed from the chirp parameters. The amplitude and phase of each discrete frequency is stored to be used later to generate the raw data from the computed scattered fields. With the view angles, satellite to target ranges, and chirp frequencies, GRECO computes the scattered monostatic EM field for each azimuth position, frequency, and polarization.

\section{GRECO Software}

GRECO is designed to predict the RCS of perfectly conducting three-dimensional (3-D) complex target models [12]-[14].

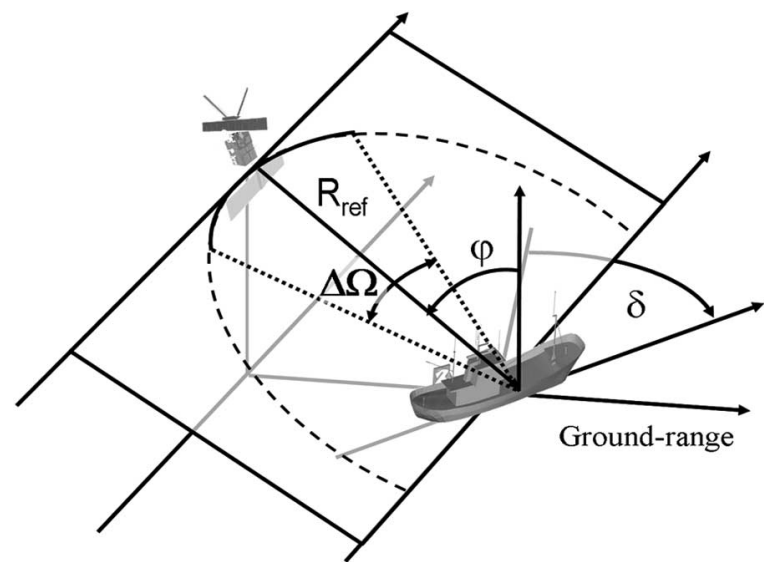

Fig. 4. Imaging geometry for POLISAR data.

It is based on high-frequency approximations that avoid the unrealistic computational requirements imposed by the discretization of Maxwell's equations when applied to electrically large models. The high-frequency methods used by GRECO are enumerated as follows.

- Physical optics (PO) for perfectly conducting surfaces.

- Method of equivalent currents (MEC) with Ufimtsev's physical theory of diffraction (PTD) coefficients or Mitzner's incremental length diffraction coefficients (ILDC) for perfectly conducting edges.

- Geometrical optics (GO) + PO ray tracing for multiple reflection analysis. Bistatic GO is used for all reflections except the last one, for which PO is used. GO divergence factors for curved surfaces are computed approximately.

GRECO can analyze models only defined by polygonal facets or parametric surfaces. Several formats are admitted as International Graphic Exchange Specification v.5.2 (IGES) or AutoCAD .dxf files. For the models presented in this paper, the UPC's CAD software package GiD [16] has been used for its design.

\section{E. Raw Data and SAR Image Generation}

To get the final raw data, it is necessary to come back to time domain via an inverse FFT in the range applied for each azimuth position to the complex scattered fields calculated by GRECO. Previously, each frequency sample has been weighted with its original amplitude and phase. Once the fully polarimetric raw data are available, the SAR processor is used to focus the images. The simulator has been radiometrically calibrated to provide the RCS normalized to the resolution cell $\sigma_{o}$.

\section{F. POLISAR Mode}

The simulator can also generate POLISAR data. It uses a circular spotlight mode in which the radar is rotating around the target at fixed distance and incidence angle (see Fig. 4) [17]. The angular aperture $(\Delta \Omega)$ can be selected from a range of angles belonging to the upper half-space defined by the sea level. Depending on the selected bandwidth and angular aperture centimetric resolutions can be easily reached, and thus, 


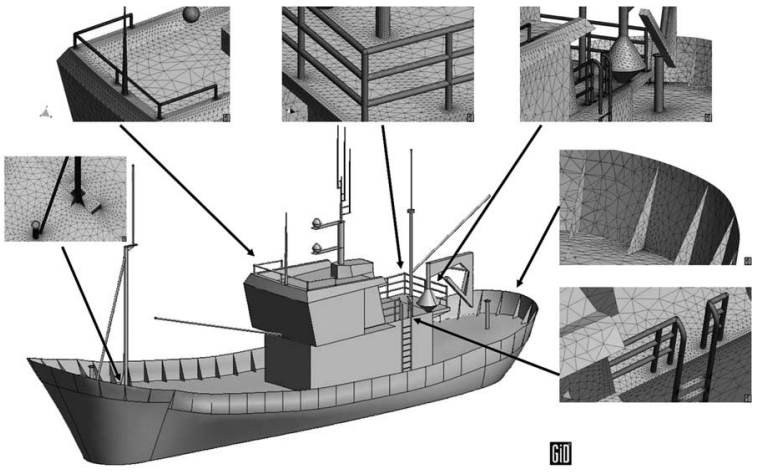

Fig. 5. Spanish fishing vessel model T1.

the obtained scattering centers and polarimetric behavior can be accurately related with target's geometry.

\section{G. Simulator Validation}

GRECOSAR has been validated using both arrays of canonical targets and complex models. The results obtained with spheres, dihedrals, and trihedrals have shown that the expected compression accuracy and polarimetric behavior is achieved. Validation with complex targets has benefited from the exhaustive comparison of the results provided by the EM solver with measurements in anechoic chambers [12]-[14]. The results have shown that the backscattered fields computed by GRECO are reliable enough as to assume that the POLSAR images generated by the simulator provide realistic information. Simulated POLISAR data have confirmed this result due to their capability to accurately relate the polarimetric behavior with the details of the vessel's structure.

Some POLISAR simulations have been made with this goal. Fig. 11 shows some examples for a C-band sensor with a bandwidth of $1 \mathrm{GHz}$ and angular excursion $\Delta \Omega=5^{\circ}$, which provides $15-\mathrm{cm}$ resolution in range and $36 \mathrm{~cm}$ in azimuth. These data have been generated for a Spanish fishing vessel $27 \mathrm{~m}$ long and $10 \mathrm{~m}$ wide modeled with $\sim 275000$ triangular facets (model T1 shown in Fig. 5). The fully polarimetric scattering maps have been analyzed with the sphere diplane helix (SDH) CTD developed by Krogager [18] (see Section III-B for further details). This decomposition uses three elementary mechanisms, namely: 1) sphere-trihedral-flat plane (odd number of reflections); 2) diplane (even number of reflections); and 3) helix (changes on the polarization of incident waves from linear to circular, which can be understood as a twodouble-bounce mechanism). The weight of each mechanism for each pixel of the image has been coded in RGB format. Besides some multireflection phenomena, all the retrieved maps indicate that only two kinds of structures located at specific parts of the vessel behave as strong scatters. The first class is the diplane interaction of the masts and banisters with the cabin surface, whereas the second one is trihedral-like, formed by the buttresses of the lateral flat banister. In both cases, the target's geometry fits with the polarimetric behavior, showing the consistency of GRECOSAR data. The simulations lasted two days using a dual-processor Athlon MP 1900 personal computer with 2 GB of RAM memory.
The usage at this point of real imagery for validation would indeed be interesting but at the same time very difficult as besides the image itself, a detailed model of the vessel and, more complicated, their exact bearing, speed, and attitude at the precise moment of the image acquisition would be necessary.

\section{Application to Vessel Classification Studies}

The usefulness of the simulator in vessel classification studies is assessed in this section. Simulated images are used to derive conclusions for matters that up to now have not been significantly studied due to the lack of reliable data. Two issues will be tackled, namely: 1) the analysis of vessel motion effects on the retrieval of useful parameters for classification, as ship length, and 2) the potentiality of CTD for basing a trustworthy classification algorithm.

\section{A. Vessel Motion Effects on Ship Length Retrieving}

1) Theory: Some basic algorithms have used ship length to coarsely classify ships, for example, distinguish a cargo from a fishing vessel [19]. The correct retrieval of this parameter is not easy as accuracy depends mainly on the ship's size compared with the available resolution, its orientation, and its motion history [20], [21]. The latter is the most worsening factor as it can cause signature distortions in the azimuth direction. Certainly, all slant-range velocities induced in the target scatters will modify their Doppler history, which is precisely the information used by SAR processors to properly focus and locate the different scatters in the azimuth direction. A point target with a radial velocity $v_{r}^{\text {scatter }}$ will have its spread function shifted in azimuth with respect to the expected location [22] and will even suffer from focusing inaccuracies. The shifting $\Delta X$ can be easily calculated from

$$
\Delta X=-\frac{|\vec{R}| v_{r}^{\text {scatter }}}{\left|\vec{V}_{\text {sat }}\right|}
$$

where $\vec{R}$ is the platform-to-target range vector, and $\vec{V}_{\text {sat }}$ is the platform velocity vector.

In the particular case of vessels, its cruising speed and the influence of the surrounding sea are translated into motions with up to seven degrees of freedom. They are divided in translational and rotational, and both can induce different radial velocities on the vessels' scatters [21]. Two kinds of effects can be observed. The simplest case is when all scatters have the same radial velocity causing "uniform azimuth shifts" of the whole spread function of the vessel and, thus, geolocation errors. This effect is observed when the ship has a unique linear motion, mainly defined by its cruising speed. Another source are the translational motions induced by the sea state, i.e., swaying, surging, and heaving, but although they can slightly modify the effective cruising speed, they can be neglected in practice [20]. In contrast, when rotational motions, i.e., pitching, rolling, and yawing, are dominant, the different scatters can have slant-range velocities that magnitude and sense depend 


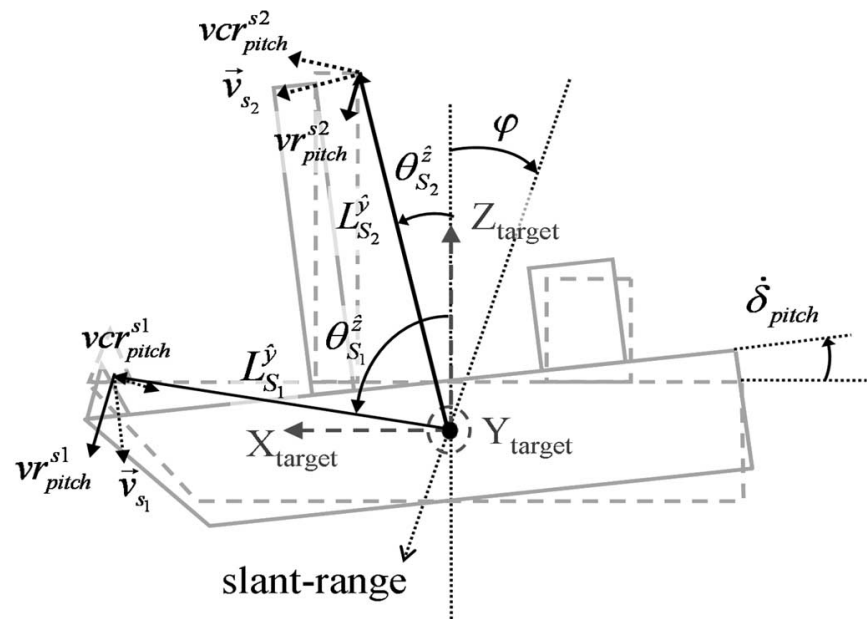

Fig. 6. Definition of the radial components for the scatters of a simple vessel affected by pitching.

on their location with respect to the rotation axes. These can result in "nonuniform azimuth shifts" when the spread function of each scatter is affected by different shifts. Consequently, the vessel's shape in the SAR image appears distorted in azimuth and its length overestimated. Normally, the heaviest distortions are observed for pitching [20], [21].

As shown in [21], it is possible to theoretically calculate the slant-range velocity for each element of the vessel's structure as a function of its location and the imaging geometry. For the $i$ th scatter, the contribution of the cruising speed is

$$
v r_{\text {speed }}^{i}=|\vec{v}| \cos (\varepsilon) \sin (\varphi)
$$

where $\varepsilon$ is the vessel bearing with respect to ground-range direction, and $\varphi$ is the incidence angle. According to the imaging geometry depicted in Fig. $2, \varepsilon=90^{\circ}-\beta-\phi$. The contributions to the radial velocity of rolling, pitching, and yawing can be approximated to

$$
v r_{\text {roll }}^{\mathrm{Si}} \cong L_{\mathrm{Si}}^{\hat{x}} \cdot \delta_{\text {roll }}\left[\sin \left(\theta_{\mathrm{Si}}^{\hat{y}}\right) \sin (\varepsilon) \sin (\varphi)-\cos \left(\theta_{\mathrm{Si}}^{\hat{y}}\right) \cos (\varphi)\right]
$$

$v r_{\text {pitch }}^{\mathrm{Si}} \cong L_{\mathrm{Si}}^{\hat{y}} \cdot \delta_{\text {pitch }}\left[\cos \left(\theta_{\mathrm{Si}}^{\hat{z}}\right) \cos (\varepsilon) \sin (\varphi)+\sin \left(\theta_{\mathrm{Si}}^{\hat{z}}\right) \cos (\varphi)\right]$

$v r_{\text {yaw }}^{\mathrm{Si}} \cong-L_{\mathrm{Si}}^{\hat{z}} \dot{\delta}_{\text {yaw }}\left[\sin \left(\theta_{\mathrm{Si}}^{\hat{x}}\right) \cos (\varepsilon)+\cos \left(\theta_{\mathrm{Si}}^{\hat{x}}\right) \sin (\varepsilon)\right] \sin (\varphi)$

where in all cases $\dot{\delta}_{\text {motion }}$ is the angular velocity of the corresponding motion expressed in radians per second, $L_{\mathrm{Si}}^{\text {axis }}$ is the shortest distance in meters from the scatter $S_{i}$ to the specific rotation axis, and $\theta_{\mathrm{Si}}^{\text {axis }}$ is the orientation angle in degrees of the scatter $S_{i}$. All these parameters are defined in the schematized ship of Fig. 6 for the case of pitching.

2) Description of Simulated Scenarios: To illustrate how the simulator can help to evaluate vessel motions effects in

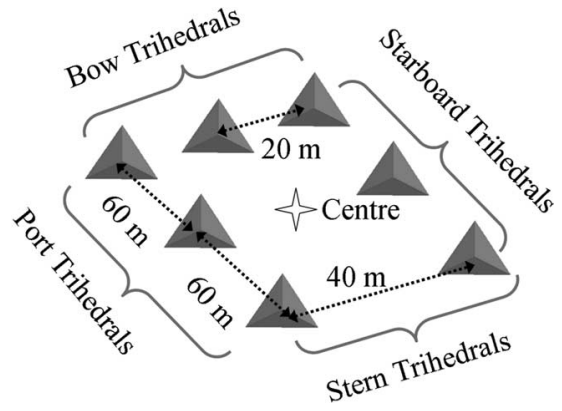

Fig. 7. Trihedral distribution of model T2. Dimensions are defined in ground range.

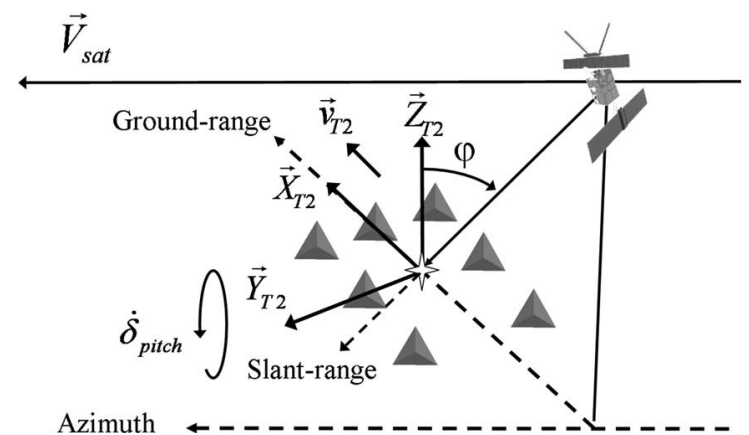

Fig. 8. Imaging geometry for the model T2.

SAR imagery and ship length retrieval, a set of simulations is presented. A simplified version of a vessel has been made using an array of seven triangular trihedrals, each with an edge length of $1 \mathrm{~m}$, faced toward the sensor. This model, labeled T2 and shown in Fig. 7, is a simple representation of the buttresses of vessel $\mathrm{T} 1$ with a trihedral-like scattering behavior. This simple distribution of scatters makes the interpretation of nonuniform azimuth shifts easier when evaluating the impact of different rotations on the vessel signature. The conclusions derived from the results can be extrapolated to more complex models without any loss of generality.

Model T2 has been simulated in four different scenarios using an ERS-like SAR sensor with null Doppler centroid. The imaging geometry, illustrated in Fig. 8, shows the vessel sailing toward the sensor (this is $\varepsilon=0^{\circ}$ ), and the angular motions have been defined according to Fig. 3. In the first simulation, labeled CALM, model T2 is static in a steady sea. In the other three, in addition to a cruising speed $v_{T 2}$ of $3 \mathrm{~m} / \mathrm{s}$, the model experiments particular rotational motions with angular velocities $\dot{\delta}_{i}$ of $0.015 \mathrm{rad} / \mathrm{s}$. The subindex $i$ indicates which rotational motion has been simulated: pitch, roll, or yaw.

3) Data Analysis: The magnitudes of the HH SAR images obtained for the four scenarios are gathered in Fig. 9 after being zero padded by a factor of 32 . As expected, the signature of simulation CALM is focused at the correct position and with the exact dimensions, while in the other three cases, the center of the target is displaced in azimuth due to cruising speed. In addition, the relative separation among the corners changes depending on the rotational motion and their position along the hull. For simulation PITCH, the distortion pattern 


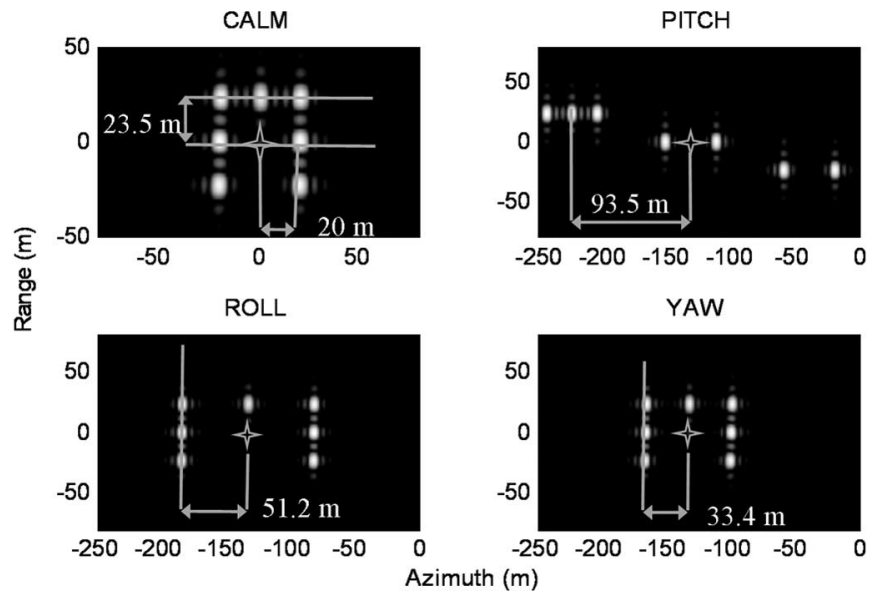

Fig. 9. Magnitudes of the interpolated HH SAR images for simulations calm, pitch, roll, and yaw.

is characterized by an azimuth shift on the location of the spread functions of the bow and stern trihedrals. Due to their equidistance with respect to the center of rotation (highlighted in Fig. 7 by a white star), the induced radial velocities have the same magnitude but opposite sense as well as azimuth shifts. The same applies for simulations roll and yaw, but not for the board and starboard trihedrals. All the shift terms observed in these images can be checked by evaluating (3)-(6) and (2) with the simulation parameters.

From the obtained results, some observations are enumerated as follows.

1) For low incidence angles $\varphi$, pitching causes the largest nonuniform shifts and azimuth length distortions. Certainly, for lower values of $\varphi$, the yaw contribution and the first terms of (3) and (4) can be neglected. This means that significant radial velocities can only be induced by those scatters located over the deck as the buttresses of the vessel T1 [21]. For pitching, these scatters can reach higher values of $L_{\mathrm{Si}}^{\mathrm{axis}}$ and, thus, induce higher radial velocities. These results corroborate those derived in [20] from the analysis of RADARSAT images.

2) Motion values have been simulated quite unrealistically to maximize azimuth shifts. Certainly, the induced radial velocity for pitching is equal to $0.9 \mathrm{~m} / \mathrm{s}$, which is far, for example, from the $0.29 \mathrm{~m} / \mathrm{s}$ retrieved in [20] for an image acquired in normal meteorological conditions. Even for a strong sea state, the estimated value is around $0.8 \mathrm{~m} / \mathrm{s}$. So, the length overestimation measured for pitching (around of $400 \%$ ) is improbable to be observed in real scenarios but not impossible. However, usual sea states can induce errors higher than $100 \%$.

3) Despite the geometric simplicity of model T2, the percentage on length overestimation can be extrapolated to a complex vessel. This can be observed in Section III-B, where SAR images for the vessel model T1 are presented for different sea states. Therefore, it can be concluded that target length is not a reliable measurement for basing a classification algorithm as it is dramatically affected by the rotational motions during image acquisition.
TABLE I

SAR SENSOR PARAMETERS FOR THE C-BAND SENSOR

\begin{tabular}{|c|c|c|c|}
\hline \multicolumn{2}{|c|}{ Platform parameters } & \multicolumn{2}{c|}{ Chirp parameters } \\
\hline Range & $\mathbf{8 5 6 1 4 7} \mathbf{~ m}$ & Freq. & $\mathbf{5 . 3} \mathbf{~} \mathbf{H z}$ \\
\hline Incidence & $\mathbf{2 3}^{\circ}$ & BW & $\mathbf{3 7 . 9 2} \mathbf{M H z}$ \\
\hline Ant. length & $\mathbf{7 . 9} \mathbf{~ m}$ & PRF & $\mathbf{2 1 5 1} \mathbf{~ H z}$ \\
\hline Speed & $\mathbf{7 5 4 5} \mathbf{~ m} / \mathbf{s}$ & FS & $\mathbf{4 6 . 2 8} \mathbf{M H z}$ \\
\hline
\end{tabular}

TABLE II

SAR SENSOR PARAMETERS FOR THE TERRASAR-X SimUlations

\begin{tabular}{|c|c|c|c|}
\hline \multicolumn{2}{|c|}{ Platform parameters } & \multicolumn{2}{|c|}{ Chirp parameters } \\
\hline Range & 554293 m & Freq. & $9.65 \mathrm{GHz}$ \\
\hline Incidence & $20^{\circ}$ & BW & $140 \mathrm{MHz}$ \\
\hline Ant. length & $4.8 \mathrm{~m}$ & PRF & $3736 \mathrm{~Hz}$ \\
\hline Speed & $7686 \mathrm{~m} / \mathrm{s}$ & FS & $156 \mathrm{MHz}$ \\
\hline
\end{tabular}

TABLE III

SCEnARio Conditions For the Simulations of SeCtion III-B

\begin{tabular}{|c|c|c|c|}
\hline Name & Bearing $\beta$ & Speed & Motion \\
\hline SD1 & $282.5^{\circ}$ & $0 \mathrm{~m} / \mathrm{s}$ & NO \\
\hline SD2 & $292.5^{\circ}$ & $0 \mathrm{~m} / \mathrm{s}$ & NO \\
\hline SD3 & $302.5^{\circ}$ & $0 \mathrm{~m} / \mathrm{s}$ & NO \\
\hline SD4 & $312.5^{\circ}$ & $0 \mathrm{~m} / \mathrm{s}$ & NO \\
\hline SD5 & $302.5^{\circ}$ & $2 \mathrm{~m} / \mathrm{s}$ & $\dot{\delta}_{\text {pitch }}=0.07 \mathrm{rad} / \mathrm{s}$ \\
\hline SD6 & $302.5^{\circ}$ & $3 \mathrm{~m} / \mathrm{s}$ & $\dot{\delta}_{\text {roll }}=0.07 \mathrm{rad} / \mathrm{s}$ \\
\hline
\end{tabular}

\section{B. Potentialities of CTD in Vessel Classification}

1) Theory: The scattering properties of deterministic complex targets are characterized by having in each resolution cell a reduced number of predominant scatters. This avoids the definition of a statistic giving no sense to the usage of distributed methods based on second-order polarimetric descriptors. The use of the Sinclair (or scattering) matrix $[\boldsymbol{S}]$ is more advisable, and in this field, CTD can be considered the best analysis method. These theorems express $[\boldsymbol{S}]$ as a complex sum of basis matrices, each of them associated to an elementary scattering mechanism. Such mechanisms can also be related to welldefined geometrical shapes. In a general formulation

$$
[\boldsymbol{S}]=\sum_{j=1}^{N} \alpha_{j}[\boldsymbol{S}]_{j}
$$

where $[\boldsymbol{S}]_{j}$ are the basis matrices, and $\alpha_{j}$ are complex numbers measuring the weight of each mechanism. The inferring of $\alpha_{j}$ for all the pixels allows the generation of a polarimetric signature map that outlines the basic features of the vessels' geometry. This property and the fact that CTD can analyze the image pixel-by-pixel, thus preserving the full resolution, seem to make these techniques suitable for complex target classification.

According to the selected basis matrices, different methods have been developed. The first approach is the Pauli theorem, which uses the Pauli spin matrices to express $[\boldsymbol{S}]$ in terms of single scattering (flat surface, sphere, or trihedral) and dihedral 


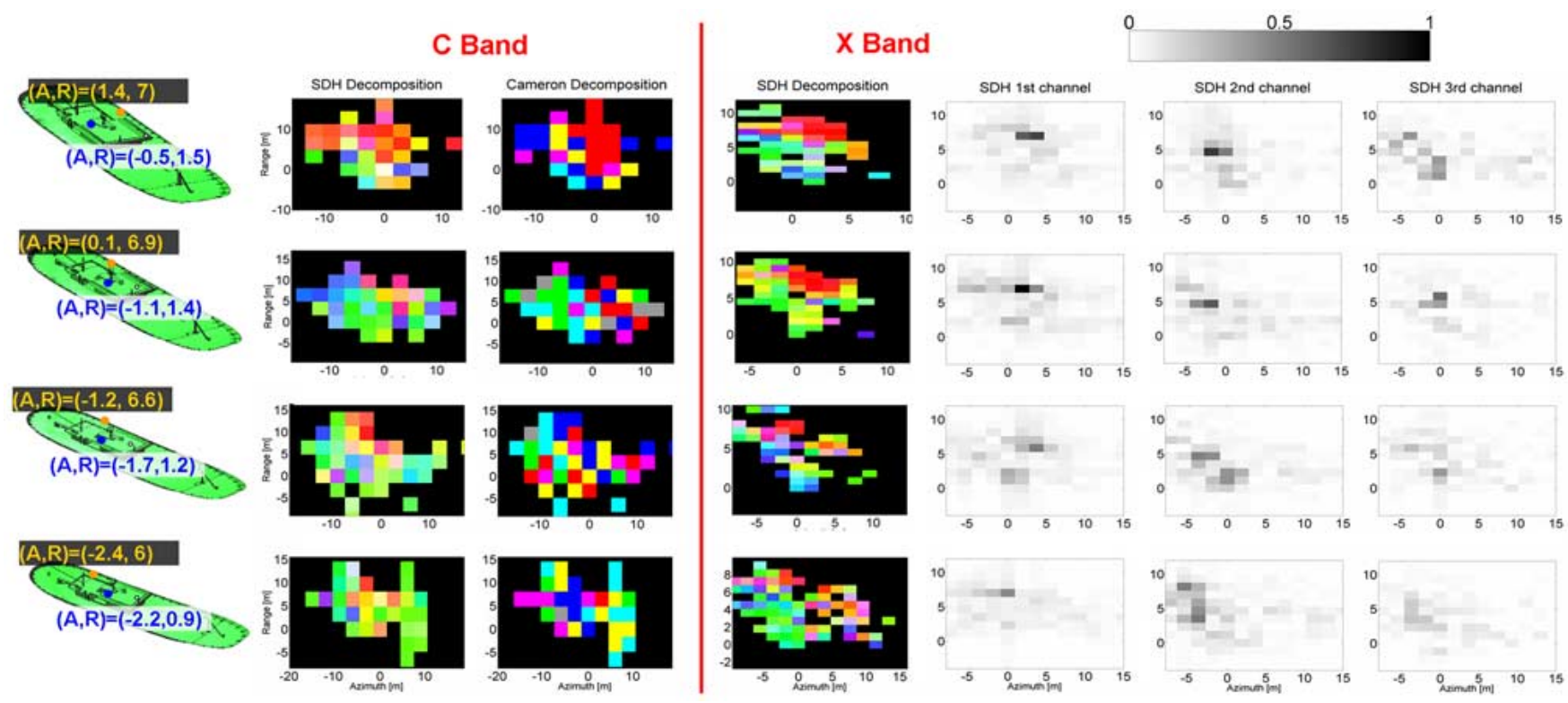

Fig. 10. Results of simulations SD1-SD4 for the C-band and X-band POLSAR sensors.
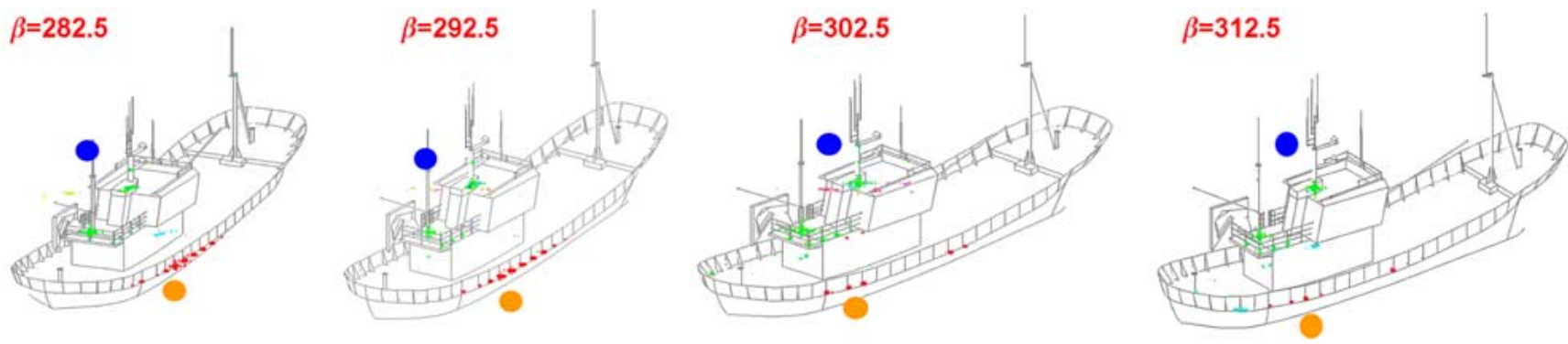

Fig. 11. SDH decomposition of POLISAR images for the C-band sensor under scenario conditions SD1-SD4.

mechanisms with an orientation of $0^{\circ}$ and $45^{\circ}$. The other approaches are the SDH developed by Krogager [18] and the Cameron one [23]. This last theorem exploits the symmetric scattering characteristics of complex targets to isolate six elemental scatters, i.e., trihedral, diplane, dipole, cylinder, narrow diplane, and quarter-wave device.

The coherent nature of CTD forces to consider all the scattering centers associated with each basis to share the same center of phase within the resolution cell. This means CTD will work properly only for those cases having a single and strong scatter per mechanism in each pixel or a reduced number of them behaving very close to the selected basis. If these conditions are not fulfilled, CTD analysis can lead to unpredictable results [24], [25]. In the following, GRECOSAR will be used to clarify the usefulness of CTD in classification.

2) Description of Simulated Scenarios: Different simulations have been generated for the vessel model presented in Fig. 5 with two different sensors, one working at C-band and the other at X-band. Table I shows the main parameters of a C-band sensor with a resolution of $3.5 \mathrm{~m}$ in both azimuth and range. Ten simulations have been made using the environmental conditions detailed in Table II. For conditions SD1 to SD4, with the vessel steady, both POLSAR and POLISAR images have been obtained, whereas with conditions SD5 and SD6, which consider the vessels moving and affected by rotations, only POLSAR has been obtained. Table III shows the sensor parameters of an X-band SAR with a resolution of $1 \mathrm{~m}$ in range and $2 \mathrm{~m}$ in azimuth. Eight simulations have been made under conditions SD1 to SD4 for obtaining both POLSAR and POLISAR images. All POLISAR simulations have a bandwidth of $1 \mathrm{GHz}$ and an angular aperture $\Delta \Omega=5^{\circ}$ that provides $15 \mathrm{~cm}$ of resolution in range and $36 \mathrm{~cm}$ in azimuth. POLISAR images have the same point of view as the POLSAR ones, which allows their comparison.

3) Data Analysis: The results of these simulations are presented in Figs. 10-12. Fig. 10 shows the POLSAR images for the $\mathrm{C}$-band and X-band sensors under simulation conditions SD1 to SD4. Results have been ordered in four rows, one for each bearing. The first column shows the vessel under the point of view of the satellite with the location of two reference points corresponding to two significant scatters. The second and third columns show, respectively, the C-band data analyzed with SDH and Cameron decompositions. The remaining columns show the results for the X-band sensor: the fourth displays the RGB image of the SDH decomposition, while the fifth, sixth, and seventh display each channel of the decomposition. 


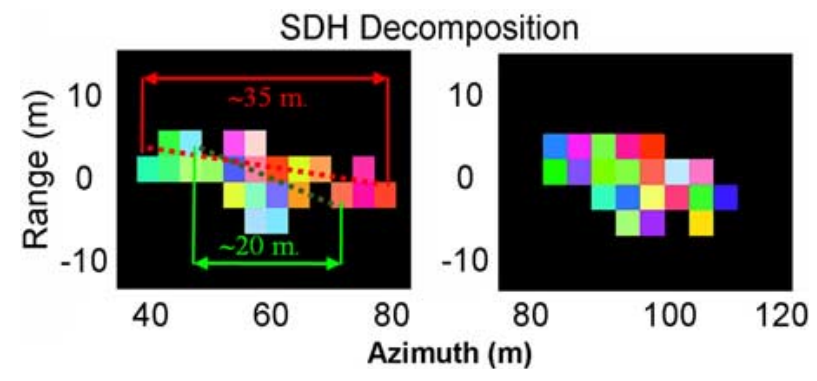

Fig. 12. Results of simulations SD5 and SD6 for the C-band POLSAR sensor.

Fig. 11 presents the RGB images derived after applying SDH to the C-band POLISAR images under conditions SD1 to SD4. Colored circles point at the most important scattering centers of the vessel, which correspond to the two reference points of POLSAR images. The POLISAR data for the X-band sensor have not been included as they are very similar to the C-band. Finally, Fig. 12 shows the RGB image derived from the application of SDH to POLSAR data simulated under conditions SD5 and SD6. All color codes are summarized in Table IV. Whereas Pauli and SDH theorems use RGB images, Cameron uses a color-coded format. This last theorem considers seven mechanisms, the six symmetric, i.e., trihedral, diplane, dipole, cylinder, narrow diplane, and quarter-wave device, and another labeled asymmetric that gathers all asymmetric contributions. Black color is used to hide those pixels with a span value under a fixed threshold.

From the obtained results, some observations are enumerated as follows.

1) The polarimetric behavior of the ship obtained with CTD from high-resolution POLISAR images is quite stable with respect to different bearings. Certainly, similar scattering mechanisms, which can be associated to welldefined parts of the vessel's structure, appear in all the images. The most important ones are the buttresses, with a trihedral-like mechanism, and the masts, with a diplane interaction. Such scatters could be the base of a classification pattern as their behavior is preserved for a wide solid angle of observation conditions. Especially important is the dihedral mechanism, which is observed for most bearings (further ISAR tests have confirmed this point).

2) On the contrary, and with the same RGB representation, the polarimetric behavior of the ship derived from SAR images is not so stable with respect to the vessel bearing than in the previous case. Although there are some pixels that could be related with the main scattering centers and the vessel's structure, the information provided by CTD is strongly dependent on the bearing. Similar results will be obtained modifying the incidence angle. Even worst are the results for simulation conditions SD5 and SD6, where the polarimetric behavior derived from CTD experiments almost randomly changes. It can also be noticed that the enlargement of the vessel's azimuth length with condition SD5 is quite similar to that retrieved in simulation PITCH
TABLE IV

COLOR CODE FOR DECOMPOSITION IMAGES

\begin{tabular}{|c|c|c|c|c|}
\hline Pauli & \multicolumn{2}{|c|}{ Single scattering } & Dihedral $0^{\circ}$ & Dihedral $45^{\circ}$ \\
\hline SDH & \multicolumn{2}{|c|}{ Sphere, Trihedral,... } & Diplane & Helix \\
\hline \multirow[b]{2}{*}{ Cameron } & \multicolumn{2}{|c|}{ Trihedral } & Dihedral & Dipole \\
\hline & Cylinder & $\begin{array}{l}\text { Narror } \\
\text { Diplan }\end{array}$ & 1/4 Device & $\begin{array}{c}\text { Anti- } \\
\text { symmetric }\end{array}$ \\
\hline
\end{tabular}

of Section III-A, but with an azimuth length overestimation of around $80 \%$.

3) If the previous analysis is performed separately on the three SDH channels, it can be found that for each bearing, a similar scattering distribution can be isolated. An accurate evaluation of the location and behavior of the predominant mechanisms shows that the derived polarimetric signature can be qualitatively linked with those retrieved in ISAR images. But the poorer resolutions of SAR images with respect to ISAR ones represent a strong limitation. Even in the case of the X-band data, better resolution is not enough. As shown in Fig. 10, it is possible to distinguish the clear trihedral-like mechanisms induced by the buttresses and the dihedral-like ones due to masts over the cabin. However, at first sight, the retrieved information seems to be not enough for a trustworthy classification.

These results confirm that a classification approach cannot be based only on CTD unless sensors supporting very high resolution modes were available. In any case, the highlighted limitations do not mean polarimetry has to be considered useless for vessel classification. Just the contrary, POLISAR and POLSAR images have shown that the polarimetric behavior of the vessel is dominated by a reduced number of significant scattering centers that preserve their polarimetric behavior for a reasonably wide range of observation conditions. The structures that generate these mechanisms are very common in most vessels; thus, it seems reasonable that similar results can be obtained for all of them. Obviously, their distribution would vary according to the particular geometrical structure of each ship, and this is the clue for allowing their classification. The only point that must be solved is how to reliably retrieve such information. In this field, it is mandatory to derive from data quantitative measurements of the target's geometry instead of the qualitative analysis provided by CTD. The relative height among scatters obtained via single-pass SAR interferometry can be a solution. If no degradation on the interferometric phase is observed, this parameter is more stable with respect to the observation conditions, like the vessel's bearing, incidence angle, and sea state. Work performed by the authors based on combining polarimetry and interferometry (POLIn-SAR) has confirmed this stability [26]. Preliminary results have shown that this kind of data can provide $3-\mathrm{D}$ distribution of scatters with a notable invariance with respect to the observation conditions. These results encourage further investigations in this field. 


\section{CONCLUSION}

A numerical tool able to provide POLSAR and POLISAR images of complex targets has been presented and validated. It is based on the UPC's EM solver GRECO that predicts with high-frequency methods the EM fields scattered by 3-D models. The simulator provides high flexibility on scenario configuration and an efficient management of the computer resources, which allows the usage of a simple personal computer to obtain significant amounts of data for electrically large models. With these features, the simulator becomes a useful tool for the development of applications requiring large amounts of data under a wide range of observation conditions and with precise ground truth. These advantages are even more crucial when the data requirements cannot be fulfilled with real measurements.

This paper assessed the usefulness of the simulator for providing the data required in vessel classification studies using SAR imagery. Simulated POLSAR and POLISAR data sets have been generated for different sensors and targets under different environment conditions. Two particular issues have been analyzed, namely: 1) the signature distortions induced by the vessels' motions during image acquisition and, especially, its impact on ship length retrieval; and 2) the suitability of CTD in classification applications. On one hand, vessel motions may cause significant distortions on the ships' SAR images that greatly drop the classification capability of image features such as ship length and bearing. On the other hand, exhaustive ISAR tests have shown that the polarimetric signature of vessels can be used as an identification map as their scattering behavior is dominated by some significant scatters that preserve their properties in a wide solid angle. Unfortunately, the analysis of POLSAR images has shown that a classification algorithm cannot be based only in CTD as the practical usage of this polarimetric tool is strongly limited by the image resolution. The discussion on CTD performance has pointed out that the next step in vessel classification should combine the benefits of polarimetry with a quantitative measure of the ship's scatters distribution. Single-pass POLIn-SAR can provide the required data for retrieving 3-D height profiles of the different scatters distributed along the vessels' structure.

The presented simulator is a powerful tool for vessel SAR classification studies, but it can also be extensively used in a wider range of SAR research areas, for instance, the design and evaluation of future missions.

\section{ACKNOWLEDGMENT}

The authors would like to thank A. Armon for providing the blueprints of the Spanish fishing vessel, A. Smith (Phoenix Systems) for useful comments on SAR image distortions, and GiD's supporting team for the free licenses.

\section{REFERENCES}

[1] J. Verborgh. (2000, Oct.). "The European VMS: An illustration of the successful introduction of advanced technologies for MCS," in Proc. Int. Conf. Fisheries Monitoring, Control and Surveillance, Brussels, Belgium, pp. 1-16. [Online]. Available: http://ec.europa.eu/fisheries/meetings_ events/events/archives/events_2000/conference_241003_en.htm

[2] G. Lemoine, "IMPAST Final Report," Deliverable of the IMPAST project with reference IMPAST/D 1.4/2.0, JRC, 2004. [Online]. Available: http://fish.jrc.cec.eu.int/fisheries/impast/deliverables.html

[3] Summary of DECLIMS Project's Goals. [Online]. Available: http:// intelligence.jrc.cec.eu.int/marine/declims/front.html

[4] S. Cloude and E. Pottier, "A review of target decomposition theorems in radar polarimetry," IEEE Trans. Geosci. Remote Sens., vol. 34, no. 2, pp. 498-518, Mar. 1996.

[5] J. J. Mallorqui, J. M. Rius, and M. Bara, "Simulation of polarimetric SAR vessel signatures for satellite fisheries monitoring," in Proc. IGARSS, 2002, vol. 5, pp. 2711-2713.

[6] G. Margarit, P. Blanco, J. Sanz, and J. J. Mallorqui, "Orbital SAR simulator of fishing vessel polarimetric signatures based on high frequency electromagnetic calculations," in Proc. IGARSS, 2003, vol. 7 , pp. 4459-4461.

[7] Y. Huang, G. Séguin, and N. Sultan, "Multi-frequency and multipolarization SAR systems analysis with simulation software developed at CSA," in Proc. IGARSS, 1997, vol. 1, pp. 536-538.

[8] G. Franceschetti, M. Migliaccio, D. Riccio, and G. Schirinzi, "SARAS: A synthetic aperture radar (SAR) raw signal simulator," IEEE Trans. Geosci. Remote Sens., vol. 30, no. 1, pp. 110-123, Jan. 1992.

[9] G. Franceschetti, M. Migliaccio, and D. Riccio, "On ocean raw data simulator," IEEE Trans. Geosci. Remote Sens., vol. 36, no. 1, pp. 84-100, Jan. 1998.

[10] S. H. W. Simpson, P. Galloway, and M. Harman, "Applications of epsilon a radar signature prediction and analysis tool," in Proc. IRS. Romsey, U.K.: Roke Manor Research Ltd., Sep. 1998. [Online]. Available: http://www.roke.co.uk/sensors/epsilon/publications.asp

[11] RadBase Data Sheet, San Diego, CA: Surface Optics Corp. [Online] Available: http://www.surfaceoptics.com/brochures/RadBase2_tech_ paper.PDF

[12] J. M. Rius et al., "GRECO: Graphical electromagnetic computing for RCS prediction in real time," IEEE Antennas Propag. Mag., vol. 35, no. 2, pp. 7-17, Apr. 1993.

[13] , "High frequency RCS of complex radar targets in real time," IEEE Trans. Antennas Propag., vol. 41, no. 9, pp. 1308-1319, Sep. 1993.

[14] — "GRECO: Graphical processing methods for high frequency RCS prediction," Ann. Telecommun., vol. 50, no. 5/6, pp. 551-556, May/Jun. 1995

[15] J. Sanz, P. Prats, and J. J. Mallorqui, "Platform and mode independent SAR data processor based on the extended chirp scaling algorithm," in Proc. IGARSS, 2003, vol. 6, pp. 4086-4088.

[16] GiD Support Database, Barcelona, Spain: Int. Center for Numerical Methods Eng. (CIMNE). [Online]. Available: http://gid.cimne.upc.es/

[17] D. R. Wehner, High Resolution Radar. Norwood, MA: Artech House, 1987.

[18] E. Krogager, "New decomposition of the radar target scattering matrix," Electron. Lett., vol. 26, no. 18, pp. 1525-1527, Aug. 1990.

[19] S. Slomka, D. Gibbins, D. Gray, and B. Haywood, "Features for high resolution radar range profile based ship classification," in Proc. ISSPA, Brisbane, Australia, Aug. 1999, pp. 329-332.

[20] K. Ouchi, M. Ichara, K. Morimura, S. Kumano, and I. Takami, "Nonuniform azimuth image shift observed in the Radarsat images of ships in motion," IEEE Trans. Geosci. Remote Sens., vol. 40, no. 10, pp. 2188-2195, Oct. 2002.

[21] G. Margarit, J. J. Mallorqui, and J. M. Rius, "Study of the vessel speed and sea swell effects on simulated polarimetric high resolution SAR images," in Proc. EUSAR, 2004, vol. 2, pp. 603-606.

[22] K. Ouchi, "On the multilook images of moving targets by synthetic aperture radars," IEEE Trans. Antennas Propag., vol. AP-33, no. 8, pp. 823-827, Aug. 1985.

[23] W. L. Cameron, N. Youssef, and L. K. Leung, "Simulated polarimetric signatures of primitive geometrical shapes," IEEE Trans. Geosci. Remote Sens., vol. 34, no. 3, pp. 793-803, May 1996.

[24] G. Margarit, X. Fabregas, and J. J. Mallorqui, "Study of the polarimetric mechanisms on simulated vessels with SAR and ISAR imaging," in Proc. EUSAR, 2004, vol. 1, pp. 447-450.

[25] G. Margarit, X. Fabregas, J. J. Mallorqui, and T. Broquetas, "Analysis of the limitations of coherent polarimetric decompositions on vessel classification using simulated images," in Proc. IGARSS, 2004, vol. 4, pp. 2483-2486.

[26] G. Margarit, X. Fabregas, J. J. Mallorqui, and L. Pipia, "Orbital polarimetric SAR interferometry simulator of complex targets," in Proc. IGARSS, 2005, vol. 3, pp. 2015-2019. 


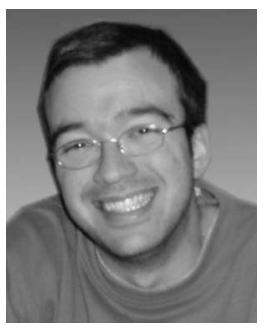

Gerard Margarit (S'05) was born in Barcelona, Spain, in 1979. He received the Diploma degree in electrical engineering and the Advanced Studies Diploma degree from the Universitat Politècnica de Catalunya (UPC), Barcelona, in 2002 and 2004, respectively. $\mathrm{He}$ is currently working toward the Ph.D. degree at UPC.

In 2004, he was a "Stagier" at the Joint Research Centre, Ispra, Italy, for a period of four months. His main research topics are three-dimensional SAR simulation of complex targets, polarimetric SAR interferometry, and vessel classification.

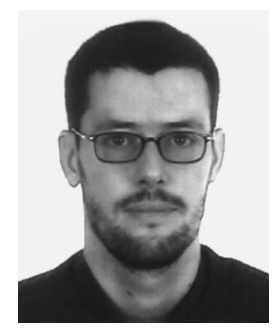

Jordi J. Mallorqui (S'90-M'96) was born in Tarragona, Spain, in 1966. He received the Ingeniero and Doctor Ingeniero degrees from the Universitat Politècnica de Catalunya (UPC), Barcelona, Spain, in 1990 and 1995, respectively, both in telecommunication engineering.

$\mathrm{He}$ was a Ph.D. student at the Department of Signal Theory and Communications in 1991, an Assistant Professor in 1993, and has been an Associate Professor at the Telecommunications Engineering School, UPC, since 1997. His teaching activity involves microwaves, radio navigation systems, and remote sensing. In 1999 , he spent a sabbatical year at the Jet Propulsion Laboratory, CA, where he worked on interferometric airborne SAR calibration algorithms. He is currently working on the application of SAR interferometry to terrain deformation monitoring with orbital and airborne data, vessel detection and classification from SAR images, and three-dimensional electromagnetic simulation of SAR systems. He is also collaborating in the design and construction of a groundbased SAR interferometer for landslide control. He is also currently developing the hardware and software of a bistatic parasitic SAR for interferometric applications using ERS and ENVISAT as sensors of opportunity. He has published more than 80 papers on microwave tomography, electromagnetic numerical simulation, and SAR processing, interferometry, and differential interferometry in referred journals and international symposia.

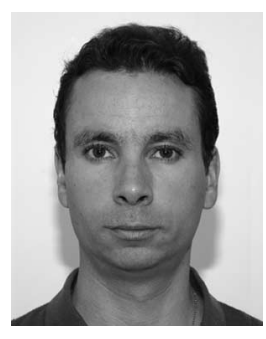

Juan M. Rius (S'89-M'91) received the Ingeniero de Telecomunicación and Doctor Ingeniero degrees from the Universitat Politècnica de Catalunya (UPC), Barcelona, Spain, in 1987 and 1991, respectively.

In 1985, he joined the Electromagnetic and Photonic Engineering Group, Department of Signal Theory and Telecommunications, UPC, where he is currently a "Catedrático" (equivalent to Full Professor). He was a Visiting Professor at the Ecole Polytechnique Fédérale de Lausanne (EPFL), Switzerland, from May 1, 1996 to October 31, 1996; a Visiting Fellow at the City University of Hong Kong from January 3, 1997 to February 4, 1997; the CLUSTER Chair at EPFL from December 1, 1997 to January 31, 1998; and a Visiting Professor at EPFL from April 1, 2001 to June 30,2001 . He has about 41 papers published or accepted in refereed international journals (17 in IEEE Transactions) and more than 100 in international conference proceedings. From 1985 to 1988, he developed a new inverse scattering algorithm for microwave tomography in cylindrical geometry systems. Since 1989, he has been engaged in the research for new and efficient methods for numerical computation of electromagnetic scattering and radiation. He is the developer of the graphical electromagnetic computation (GRECO) approach for high-frequency RCS computation, the integral equation formulation of the measured equation of invariance, and the multilevel matrix decomposition algorithm (MLMDA) in three dimensions. Current interests are the numerical simulation of electrically large antennas, multiband fractal antennas, large microstrip arrays, and reflector antennas.

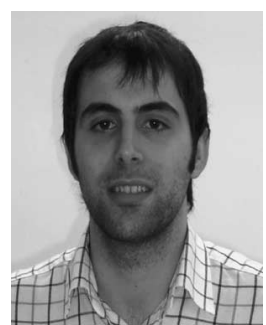

Jesus Sanz-Marcos was born in Barcelona, Spain, in 1979. He received the Engineer degree in telecommunication and the Advanced Studies Diploma degree from the Universitat Politècnica de Catalunya (UPC), Barcelona, in 2002 and 2004, respectively.

From 2000 to 2002, he was with Aplicaciones en Informatica Avanzada, working in San Francisco, Rio de Janeiro, and Mexico, where he analyzed power network systems. From 2002 to 2005, he was a Ph.D. student at the Signal Theory and Communications Department, UPC. Since 2006, he has been with Indra Espacio, Remote Sensing Department, UPC, where he performs research in the SAR field. His main research is devoted to the development of bistatic fixed received SAR systems. 